\title{
II.
}

\section{Zur Kenntniss der Tractions-Divertikel des Oesophagus.}

\author{
Von \\ Prof. Dr. Hugo Ribbert in Marburg.
}

Die Entstehung der Tractions-Divertikel, die überwiegend an der vorderen Wand des Oesophagus unterhalb der Bifurcation der Trachea vorkommen, eine trichterförmige Gestalt haben und schräg nach oben gerichtet sind, wird heute allgemein auf die narbige Schrumpfung von Entzündongs-Heerden zurückgeführt, welche aussen an einer umschriebenen Stelle der Speiseröhre sassen und mit ihrer Wand in Verbindung getreten waren. In weitaus den meisten Fällen sah man tuberculös-anthrakotische Lymphdrüsen direct oder durch Vermittelung eines Narbenstranges in Zusammenhang mit der Spitze des Divertikels, dessen Schleimhaut in der Tiefe häufig narbige Veränderungen aufwies. Demgemäss stellt man sich gewöhnlich vor, dass die zunächst an den Oesophagus angehefteten und erweichten Drüsen in das Lumen durchbrachen, und dass dann ihre unter Schrumpfung erfolgende Ausheilung jenen Zug ausübte. Doch ist eine Perforation nicht unbedingt erforderlich. Denn eine entzündlich vergrösserte und angewachsene Drüse kann ja auch ohne Entleerung ihres Innern eine narbige Verkleinerung eingehen.

So einleuchtend nun diese Genese ist, so wenig ist sie doch frei von Bedenken.

Die Tractions-Divertikel finden sich hauptsächlich bei älteren Leuten. Wenn nun wirklich die Verwachsung, Erweichung und Perforation von Lymphdrüsen jene Bedeutung hätte, so sollte man doch bei jüngeren Leuten mindestens ebenso häufig, - in Wirklichkeit aber häufiger, da ja nicht alle solche Processe in Divertikel-Bildung auszugehen brauchten, - jene Verlöthungen oder jene Durchbrüche antreffen. Aber das ist nicht der Fall, vielmehr sind solche Befunde äusserst selten. Ich selbst habe keinen derartigen Fall gesehen, habe aber sehr viele Divertikel beobachtet, die ja gar nicht selten sind. Man könnte auch nicht etwa einwenden, dass jene einleitenden Vorgänge zu rasch ab- 
liefen. Denn die Anheftung der Lymphdrüse und die Perforation bis zum Beginn einer narbigen Retraction müssten sehr langsam vor sich gehende Veränderungen darstellen und demgemäss sehr oft gesehen werden. Das beweisen uns die analogen Processe an den Bronchen, der Trachea und besonders den Pulmonalgefässen. An diesen Gebilden beobachten wir eine feste Vereinigung mit Lymphdrüsen sehr oft und nicht selten auch eine Perforation in das Lumen. Aber wir sehen hier kaum jemals eine trichterförmige Ausziehung zu Stande kommen, die den Tractions-Divertikeln des Oesophagus entsprechen würde.

Diese Ueberlegungen allein zeigen zur Genüge, dass für die Genese der Divertikel die schrumpfende Lymphdrüse nicht das allein maassgebende sein kann.

Dazu kommt noch, dass die angenommene Verlöthung mit dem Oesophagus keineswegs so leicht verständlich ist, wie es bei der Häufigkeit der Divertikel vorausgesetzt werden müsste. Denn die bronchialen und trachealen Lymphdrüsen haben zur Speiseröhre keine directe Beziehung, sie sind durch reichliches, lockeres Bindegewebe von ihr getrennt. Es ist deshalb etwas ganz Anderes, ob die Drüsen, wie es ja sehr oft geschieht, mit den Bronchen oder den Pulmonalgefässen, oder ob sie mit dem Oesophagus verwachsen. Thatsächlich bleiben ja auch die tuberculös anthrakotischen Veränderungen der Lymphknoten meist ohne Beziehung zum Oesophagus. Es muss also noch ein be. sonderer Umstand in Betracht kommen, der die Anheftung eines Narbenstranges oder einer narbigen Drüse an die Divertikel erklärt.

Ein weiteres Bedenken liegt in der Thatsache, dass nicht selten Divertikel vorkommen, die, bei typischer Ausbildung, keinerlei Zusammenhang mit Entzïndungs-Heerden, insbesondere mit schrumpfenden Drüsen zeigen, die also ganz unabhängig von solchen entstanden sein müssen.

In der Literatur haben diese Fälle keine ausreichende Beachtung gefunden. Man hat aus ihnen keine Einwände gegen die herrschende Lehre entnommen, hat sich auch überhaupt nur wenig mit ihnen beschäftigt. Aber sie veranlassten mich, im Verein mit den anderen Ueberlegungen, den Tractions-Divertikeln meine Aufmerksamkeit zuzuwenden. 
Ich sammelte aber nicht nur solche Präparate, in denen die Ausbuchtung sofort ins Auge fiel und voll entwickelt war, sondern auch weniger weit ausgebildete, in denen nur kleine; trichterförmige und flache Gruben zu sehen waren. Derartige Divertikel können bei der Ausbreitung des aufgeschnittenen Oesophagus leicht mehr oder weniger verstreichen, und deshalb unter Umständen übersehen werden.

Von 40 Divertikeln, die bis auf eines, oberhalb der Bifurcation gelegenes an der typischen Stelle unterhalb der Trachea sassen und schräg gegen sie nach aufwärts gerichtet waren, besitze ich mikroskopische Präparate, die in der Längsrichtung des Oesophagus angelegt sind. Die an ihnen beobachteten Verhältnisse stelle ich in der Weise zusammen, dass ich mit den am wenigsten ausgebildeten beginne und zu denen fortschreite, welche das Divertikel in der bekannten $W$ eise mit anthrakotischen Processen im Zusammenhang zeigen.

1. Die einfachste Form ist durch eive flache SchleimhautEinsenkung gegeben, der Nichts weiter entspricht, als ein relativ breites, intermusculäres, bindegewebiges Interstitium, in welchem Gefässe aus der Umgebung des Oesophagus zur Schleimhaut hindurchtreten. Eine Beziehung zur Divertikel-Bildung wird nur dadurch sichtbar, dass man die Wand der unteren SpeiseröhrenHälfte nach abwärts zieht, während der obere Theil und die Trachea fixirt sind. Dann hält jener gefässhaltige Strang die Schleimhaut fest und verursacht in ihr eine leicht trichterförmige Einsenkung. Ich besitze zwei derartige Objecte.

2. Drei weitere Präparate lassen jenen Bindegewebs-Zug nicht mehr als ein breiteres Interstitium, sondern als eine deutliche Lücke in den Muskelschichten, als eine ausgesprochene Unterbrechung erscheinen. In zweien dieser Fälle setzte sich das eingeschaltete Gewebe in Form eines gut umgrenzten bindegewebigen Zuges nach aufwärts gegen die Trachea fort. Der eine der beiden Züge geht dicht an einer kleinen, pigmentirten, sonst aber unveränderten Lymphdrüse vorüber. In dem dritten Object ist das Bindegewebe jener Lücke ausserordentlich gefässreich und findet ebenfalls in einem, aber weniger gut geschlossenen Zuge seine Fortsetzung. 
Die eigentliche Divertikel-Bildung ist an diesen drei Speiseröhren noch wenig ausgeprägt. Das Epithel erscheint flach ausgebuchtet oder es bildet im Bereich der Muskellücke eine kleine, trichterförmige Einsenkung.

$J_{e}$ ausgedehnter aber die Unterbrechung der Muskelschichten ist, um so leichter combinirt sich mit ihr eine weitergehende Ausbuchtung.

3. In einem derartigen Präparat ist eine dreifache Lückenbildung vorhanden, die einem gemeinsamen, flach concaven Bezirk angehört. Aber jeder der drei Stellen entspricht eine besondere kleine Einsenkung des Epithels.

4. In drei weiteren Objecten reicht die trichterförmig sich einsenkende Vertiefung etwas über die äussere Muskellage hinaus. Sie geht schräg nach oben und verlängert sich in einen parallel gefaserten, von der Umgebung durch dichteres, aber keineswegs narbiges Gefüge sich abhebenden, bindegewebigen Strang, der eine Strecke weit za verfolgen ist. Ring- und LängsMusculatar schneiden am Rande des Divertikels scharf und ohne weitere Veränderung ab.

5. Wiederum drei neue Divertikel zeichnen sich durch ihre relativ beträchtliche Tiefe und typische Anordnung aus. Sie werden gebildet von dem Epithel, der Muscularis mucosae und etwas Bindegewebe, und treten so ausgestattet durch eine breite Unterbrechung der beiden Muskellagen hindurch, welche in dem einen Falle scharf abgesetzt ist, während in dem zweiten die Längsmusculatur eine Strecke weit auf die Aussenfläche des Divertikels übergeht. In dem dritten Präparat ist die Fortsetzung in einen bindegewebigen Strang ganz besonders deutlich. Er zieht von der Spitze des Divertikels fast $1 \mathrm{~cm}$ weit in regelmässtger, parallel-faseriger, lockerer Anordnung gegen die Trachea hin, lässt sich bis zu ihr, wenn auch nicht mehr ganz so geschlossen, verfolgen und inserirt am Perichondrium. In dem Zuge verlaufen in gleicher Richtung sehr dünnwandige Gefässe und Nerven. Ausserdem setzt sich in ihn die Muscularis mucosae eine längere Strecke weit, allmählich abnehmend, fort. Der Strang hat nirgendwo narbigen Charakter. Neben ihm liegen in der Nähe der Trachea mehrere kleine, pigmentirte Drüsen in wellig gefaserter Umgebung, und fast $1 \mathrm{~cm}$ rom Oesophagus 
entfernt, ebenfalls neben dem Zug, vier, mit blossem Auge kaum sichtbare, gelbliche Kalkknötchen, in lockeres Bindegewebe eingeschlossen. Ceber ihre Herkunft lässt sich Nichts aussagen. Das Epithel des Divertikels stösst hier und da an eine dünne Zone zellig infiltrirter Mucosa.

6. Eine weitere Gruppe ist dadurch gekennzeichnet, dass die Muskelschichten im Bereich des Divertikels nicht völlig unterbrochen sind, sondern in wechselnder Ausdehnung an der Ausbuchtung Theil nehmen.

Eines dieser 0 bjecte zeigt unterhalb des wenig entwickelten Divertikels lediglich eine unregelmässig angeordnete, gleichsam durcheinander gewirrte Musculatur, sonst aber keine besonderen Eigenthümlichkeiten.

In einem anderen Falle findet sich ein plattes, aber $1 \mathrm{~cm}$ weit hinaufreichendes, breites, spaltförmiges Divertikel. Es liegt zwischen der Muscularis mucosae und der Ring-Musculatur und ist mit dickem Platten-Epithel ausgekleidet. Von seinem oberen Rande setzt sich durch eine kleine Lücke der Muskellagen ein feiner, epithelhaltiger, nur mikroskopisch wahrnehmbarer Canal fort, der ausserhalb der Längsschicht im peri-oesophagealen Bindegewebe noch etwa $1 \mathrm{~cm}$ weit in schräger Richtung zu verfolgen ist und dann blind endet. In seiner Umgebung ist die Bindesubstanz dichter, als sonst, aber durchaus ohne narbigen Charakter.

In zwei weiteren Divertikeln mit flacher Ausbuchtung sind beide Muskelschichten zu erkennen, aber sie sind im Umfange der Ausbuchtung stark verdünnt. Die relativ geringe Tiefe der Tasche lässt diese verminderte Dicke der Musculatur nicht etwa als eine secundäre Dehnungs-Erscheinung auffassen. Sie muss vielmehr vor Zustandekommen des Divertikels vorhanden gewesen sein. Das gilt auch für die folgenden sieben Objecte.

In ihnen fehlt die Ring-Musculatur im Bereich der Einstülpung ganz. Sie ist an der Grenze gegen die Bucht scharf abgesetzt. Die Längs-Musculatur dagegen geht um das bald flachere, bald tiefere Divertikel herum. Einmal ist sie dabei nur wenig reducirt, in den übrigen Fällen aber deutlich vermindert, zuweilen bis auf einige wenige isolirt verlaufende Fasern. In vier von den sieben Präparaten geht von der Spitze des Divertikels ein schmaler, nicht narbiger Bindegewebs-Zug, der 
die Muskel-Elemente der Längsschicht in geringem Umfange eine kurze Strecke weit in sich aufnimmt, gegen die Trachea in die Höhe.

In den 23 bis jetat besprochenen Divertikeln besteht also keine directe Beziehung zu Entzündungs-Processen oder deren Residuen. Es ist völlig ausgeschlossen, dass ein Narbenzug für die Bildung der Ausbuchtung anzuschuldigen wäre. Dieses Resultat liess sich übrigens auch schon bei makroskopischer Betrachtung vorwegnehmen. Ich habe notirt, dass in keinem Falle eine Verwachsung mit einer geschrumpften und indurirten Lymphdrüse vorhanden war.

Unter solchen Umständen bleibt aber nur die Möglichkeit, die Entstehung der Divertikel aus einer Entwickelungs-Störung abzuleiten. Darauf ist bisher verhältmissmässig wenig Werth gelegt worden, und zwar, wie ich annehme, besonders deshalb, weil mau sein Augenmerk hauptsächlich auf die mit narbigen Processen in Verbindung stehenden, weniger auf die nicht so ausgeprägten und deshalb nicht so auffallenden Formen richtete.

Klebs ${ }^{1}$ ) hat zuerst hervorgehoben, dass Störungen in der Entwicklung des Oesophagus und der Trachea zu DivertikelBildung führen könnten, und Eppinger ${ }^{2}$ ) hat sich dieser Meinung angeschlossen. Beide verweisen auf die seltenen Fälle, in denen eine Fistelbildung zwischen dem Oesophagus und der Trachea, die beide im Uebrigen gut ausgebildet sind, in der Höhe der Bifurcation besteht. Eppinger führt zwei derartige Beobachtungen aus der Literatur an. Starck ${ }^{3}$ ) weist auf einen dritten von Tarnier mitgetheilten hin, in welchem eine $2 \mathrm{~cm}$ lange, spaltförmige Communication bestand.

Klebs und Eppinger ziehen ferner die nicht so sehr seltene Missbildung heran, bei welcher der Oesophagus in seiner Mitte unterbrochen ist und von oben her nach kurzem Verlauf blind endet. Der untere vom Magen heraufsteigende Abschnitt ist entweder ebenfalls verschlossen und mit dem oberen Theil durch

1) Handbuch der patholog. Anatomie I, S. 164.

2) Ib. Larynx und Trachea S. 254.

3) Die Divertikel der Speiseröhre. Monogr. 1900. 
einen bindegewebigen Strang verbunden, oder er mündet an der Bifurcation* in die Trachea.

Alle diese Missbildungen lassen sich, wie es Eppinger thut, aus der Entwicklung ${ }^{1}$ ) von Trachea und Oesophagus ableiten. Das ursprünglich gemeinsame Rohr spaltet sich durch Bildung eines Septums in zwei Canäle. Reicht die Scheidewand, wie Klebs annahm, in der Höhe der späteren Bifurcation zu weit nach rückwärts, entwickelt sie sich auch aus der hinteren Wand des Oesophagus, so wird dieser quer abgeschlossen. Seine untere Hälfte wird dabei entweder auch verengt oder sie bleibt in Verbindung mit der Trachea. Ist die Septum-Bildung aber unvollkommen, so entsteht jene Fistel. Eine noch geringfügigere Entwicklungs-Störung kann dann die Divertikel-Bildung herbeiführen.

Auch Starck ist der Meinung, dass einzelne TractionsDivertikel auf embryonale Anomalien zu beziehen sein dürften.

Ich gehe weiter und spreche mich dahin aus, dass die Tractions-Divertikel überhaupt in ihrer weitaus grössten Zahl aus Entwicklungs-Störungen abzuleiten sind, die darin bestehen, dass in der Scheidewand zwar keine Oeffnung bleibt, aus der später ein Fistelgang werden könnte, dass aber an entsprechender Stelle eine mangelhafte Ausbildung der Oesophagus-Wand zu Stande kommt. Sie ist dadurch gekennzeichnet, dass sich die Musculatur in der Bifurcations-Höhe der Trachea an umschriebener Stelle nur unvollkommen oder gar nicht anlegt, dass an ihre Stelle Bindegewebe tritt, welches sich in einem gegen die Trachea verlaufenden und zuweilen an ihr angehefteten Zuge fortsetzt. In ihm würde in jenen seltenen Fällen der epitheliale Fistelcanal verlaufen.

Eppinger hebt hervor, "dass häufiger, als man glaubt, diverticulöse Ausstülpungen des Oesophagus (inclusive ihrer

1) Herr Geheimrath Gasser hatte die Freundlichkeit, mir die von Herrn Prof. Zumstein über die Entwicklung der Speise- und Luftröhre verfertigten ausgezeichneten Plattenmodelle zu demonstriren. Man kann an ihnen die Bildung des von der Abgangsstelle der Trachea nach oben steigenden und zugleich aus der linken und rechten Wand sich entwickelnden Septa ausgezeichnet verfolgen und sich jene Missbildungen leicht verständlich machen. 
Muskelschicht) sich mit ihrem spitzen Ende an die Bifurcationsstelle der Trachea inseriren.

Die Divertikel-Bildung kann dann auch eutweder so gedacht werden, dass die muskelfreie Stelle der Wand sich durch den Innendruck ausbuchtet, dass also Pulsions-Divertikel entstehen, die ja auch im Verlaufe des Oesophagus zuweilen beobachtet werden; oder es handelt sich, wie in den meisten Fällen, um ein Tractions-Divertikel, welches durch die Anspannung jenes Bindegewebe-Stranges zu Stande kommt, wenn der Oesophagus sich durch Wachsthum nach abwärts verlängert oder bei dem Schluck-Act etwas gegen den Magen hin verschiebt und durch Contraction von der Trachea entfernt. Je nach der festeren oder weicheren Beschaffenheit des bindegewebigen Zuges, an dem Musculatur und Schleimhaut fixirt sind, wird dann das Divertikel mehr oder weniger ausgeprägt werden.

Pulsions- und Tractions-Divertikel können sich aber auch combiniren, und zwar besonders in der Weise, dass ein durch Zug auf die eben erwähnte Weise entstandenes oder auf die noch zu besprechende Weise vertieftes Divertikel sich durch den Druck von innen her noch weiter ausbuchtet. Oekonomides ${ }^{1}$ ) hat auf diese Möglichkeit hingewiesen.

Die Entwicklungs-Geschichte eröffnet uns also ein Verständniss jener Form von Tractions-Divertikeln, bei denen Entzündungs-Processe auszuschliessen sind. Dahin gehören die 23 zuerst beschriebenen Präparate.

Aber auch für die meisten übrigen Divertikel können wir die durch Entwicklungs-Störungen gegebene Grundlage nicht entbehren.

Gewiss soll nicht geleugnet werden, dass allein durch Schrumpfung eines mit der Oesophaguswand verwachsenen Entzündungs-Heerdes ein Divertikel erzeugt werden kann, aber wenn nicht für alle, so doch für die meisten an typischer Stelle, d. h. in der Gegend der Bifurcation sitzenden Ausbuchtungen glaube ich diese Entstehungsweise ausschliessen zu sollen. Denn die anatomischen Verhältnisse sind nicht so, wie es bei primärer Verwachsung oder bei einem Durehbruch von Lymphdrüsen mit nachfolgender narbiger Retraction erwartet werden müsste.

1) Dissertation, Basel 1882 . 
Nach Perforation einer Drüse und nach Entleerung des erweichten lnbaltes würde das schrumpfende Gewebe an dem äber der Oeffnung sich wieder schliessenden Epithel angeheftet sein und es sammt der gleichfalls fixirten Musculatur in die Tiefe ziehen. In ähnlicher Weise würde der Zug wirken, wenn nur eine entzündliche Infiltration der Musculatur bis zum Epithel zu Stande gekommen wäre, während bei einer nur mit der äusseren Muskelschicht bestehenden Verlöthung zunächst allein diese Lage, secundär aber auch die Ringmusculatur und das Epithel ausgebuchtet werden müssten.

Nun besitze ich aber nur 4 Divertikel, welche diesen Anforderungen einigermaassen entsprechen würden. Aber auch bei ihnen ist eine andere Erklärung möglich und wahrscheinlicher, welche nicht ausschliesslich auf die Narben-Retraction Bezug nimmt. Ich bespreche sie zuletzt und gehe zunächst anf andere Divertikel ein.

7. In fïnf makroskopisch typischen Präparaten findet sich zwar ein in der Nähe der Ausbuchtung nachweisbarer, mehr oder weniger derber, narbiger, nach aufwärts strebender, mit etwas Kohle-Einlagerung versehener Zug, aber er heftet sich nicht so an den Oesophagus an, wie es nach jever Voraussetzung sejn müsste. Unterhalb des Epithels, an der Seite oder Spitze des Divertikels, liegt zunächst eine Schicht lockeren, zweifellos vicht entzündlichen Bindegewebes. Es geht erst in einiger Entfernung in den Strang über, der seinerseits nicht ein beliebig angeordnetes narbiges, sondern ein in der Längsrichtung parallelgefasertes Gewebe aufweist. Eine ausgesprochene Beziehung zu geschrumpften Lymphdrüsen ist nicht vorhanden. In drei Fällen lag hinter dem Oesophagus nach oben von dem Divertikel je eine pigmentirte Lymphdrüse, aber ohne Induration und ohne Verlöthung mit dem nahe vorüberziehenden Strang. In einem vierten Falle war keine derartige Drüse vorhanden, und im fünften lag in dem Strang ein kleines Kalkknötchen als Rest eines alten, mit Nekrose verbundenen Entzündungs-Heerdes. Längs- und RingMusculatur war in diesen fün Präparaten um den Eingang in das Divertikel scharf abgesetzt oder ging ein wenig auf seine Seitenfläche über.

Sehen wir von der narbigen Beschaffenheit jenes Stranges 
$a b$, so liegen die Verhältnisse in den fünf Objecten so, wie in den oben zuletzt beschriebenen, in denen jeder Narbenstrang vermisst wurde. Wir brauchen nur anzunehmen, dass zu diesen, nur auf entwicklungsgeschichtlicher Basis zu deutenden Ausstülpungen ein tuberculös-anthralsotischer Process hinzukam, um die beschriebenen Verhältnisse zu Stande kommen zu lassen.

8. Ein weiteres Präparat zeigt entsprechend einer flacheren Ausbuchtung eine breite, durch welliges, ziemlich dichtes, aber nicht narbiges Bindegewebe ausgefüllte Muskellücko, unter der ein entzündlich verdichtetes, mit etwas Kohle durchsetztes, breit nach aufwärts ziehendes Gewebe liegt. In jener flachen Bucht hat das Epithel noch wieder eine kleine, trichterförmige Einsenkung, die, ebensowenig wie die Muskellücke, aus einem Narbenstrang abgeleitet werden kann.

9. Wieder ein anderes Object lässt eine Unterbrechung der Musculatur durch ein welliges, nicht narbiges, leicht kohlehaltiges Bindegewebe erkennen, welches bis dicht unter das Epithel des kleinen, trichterförmigen Divertikels reicht und auch an seiner Seite noch etwas in die Höhe zieht. Aber es hat keinen narbigen Charakter. Auch war makroskopisch keine Spur eines Narbenstranges zu entdecken.

10. Das nächste Präparat enthält eine breite Muskellücke, die durch deutlich narbiges Gewebe ausgefüllt ist. Er setzt sich auch nach aussen und oben fort und enthält etwas Kohle. In das Narbengewebe ragt tief, bis hart an einen Trachealknorpel, ein canalförmiges, epitheliales Divertikel hinein. Es steckt ringsum im Narbengewebe, kann also nicht durch dessen Zug bedingt sein. Der Befund wird am besten durch die Annahme verständlich, dass sich am ein bereits bestehendes Divertikel eine Entzündung ausbreitete.

11. In einem ferneren Fall war ein tiefes, schräges, $1 \mathrm{~cm}$ weit sondirbares, enges Divertikel vorhanden. Es tritt durch eine schmale Muskellücke in das Bindegewebe, biegt dann aussen von der Muskellage und parallel zu ihr nach oben um und endet in einem zellig infiltrirten, lymphatischen, kleinen Bezirk, der eine unebene, zackig begrenzte Höhle besitzt und unvollkommen mit Epithel ausgekleidet ist. In dem umgebogenen Theil wird der Canal, ausserhalb des zelligen Heerdes, von 
Narbengewebe dicht umgeben, innerhalb der Musculatur dagegen von lockerem, parallel gefasertem Bindegewebe. Dieser Umstand, die Enge der Lücke in den Muskelschichten und die scharfe Absetzung der letzteren in ihr, schliesst die Möglichkeit aus, dass ein Narbenzug die Divertikel-Bildung vorursacht hätte. Auch hier muss zu der bereits vorhandenen Ausbuchtung die Entzïndung hinzugetreten sein.

12. Ein weiteres Präparat zeigt ein schräges Divertikel, welches durch eine relativ enge Muskelöffung hindurchtritt, sich dann in Narbengewebe einsenkt, von ihm rings begrenzt wird und in einer fetzigen, von rundzellig infiltrirtem Gewebe umgebenen Höhle sein Ende findet. Das Narbengewebe setzt sich nach oben in einen Zug fort. Auch hier ist, da im Bereich der Muskellücke jede narbige Umwandlung des Bindegewebes mangelt, und da beide Muskelschichten scharf abgesetzt sind, an eine Entstehung des Divertikels aus einem primären Narbenstrang nicht zu denken.

13. Etwas abweichend ist ein Fall, der sich hauptsächlich als Pulsions-Divertikel kennzeichnet. Es bildet eine halbkugelige Ausbuchtung, der eine breite Unterbrechung der Muskelschichten entspricht, aber neben dem Epithel umfasst es auch die Muscularis mucosae. Am oberen Rande ist in einem kleinen Bezirk narbiges, kohlehaltiges Gewebe vorhanden, welches die Muscularis mucosae durchsetzt hat. Es liegt aber dem Divertikel nur an. Von einem Zug ist keine Rede.

14. Günstiger für die herrschende Anschaung scheinen die Verhältnisse in den letzten vier Divertikeln zu liegen.

Das eine besitzt eine breite Muskellücke, in welche eine entsprechend grosse Ausbuchtung sich bis fast an den äusseren Rand der Längs-Muskelschicht einsenkt. Unter dem Epithel beginnt ein dichtes, narbiges, mit Kohle mässig durchsetztes Bindegewebe, welches nach aufwärts in einen Narbenstrang übergeht. Dass dieses aber allein durch eine Retraction das Divertikel erzeugt hat, ist deshalb nicht wahrscheinlich, weil die Musculatur im ganzen Umfange der Ausbuchtung ohne jede Umbiegung und ohne jeden Uebergang in das Narbengewebe scharf absetzt.

Ein zweites, grosses und tiefes Divertikel zeigt in seiner ganzen Circumferenz ausgesprochen narbiges Bindegewebe, dem 
das Epithel unvermittelt aufsitzt. Es geht nach oben in einen Narbenstrang über. Aber auch in diesem Fall ist die Musculatur rings um das Divertikel ohne jede Beziehung zum Narbengewebe scharf begrenzt. Das wäre aber hier wie dort unmöglich, wenn ein Entzündungs-Process die Muskelschichten durchsetzt hätte. Aehnlich waren die Verhältnisse eines dritten Präparates. Es unterscheidet sich aber von den vorhergehenden dadurch, dass unter dem Epithel zunächst eine Lage nicht narbigen Bindegewebes vorhanden ist.

Am meisten verwerthbar für den primären Narbenzug ist das letzte Divertikel; welches typisch trichterförmig gebaut ist. In seiner Spitze und etwas auf seine Seitenwände übergreifend entwickelt sich ein Narbenzug, der sich nach aufwärts fortsetzt. Die Musculatur biegt an die Seitenflächen des Divertikels um. -

Ueberblicken wir nun die zuletzt beschriebenen, durch narbig entzündliche Veränderungen ausgezeichneten Divertikel, so lassen sich die anatomischen Verhältnisse der meisten von ihnen mit einem primären Narbenzuge nicht vereinigen, bei anderen sind Zweifel berechtigt, und das letzte passt sich der Vorstellung einer Entstehung durch entzündliche Retraction an.

Aber alle diese Divertikel, auch das letzte, werden uns gleichmässig und leicht verständlich, wenn wir annehmen, dass sie schon congenital angelegt und mehr oder weniger weit ausgebildet waren, als an ihrer Spitze gegen die Trachea hin ein entzündlicher Process einsetzte, der zur Bildung eines narbigen Stranges führte und secundär durch Schrumpfung die bestehende, auf die oben besprochene Weise entstandene Ausbuchtung vergrösserte. So bedeutet die zweite Gruppe der Divertikel nur eine Entwicklung und Modification der ersten.

Gegen diese Auffassung scheint aber auf den ersten Blick ein Einwand möglich. Weshalb nehmlich soll die Verlöthung eines Entzündungs-Heerdes fast allein bei Gegenwart eines congenitalen Divertikels oder einer Divertikel-Anlage, und weshalb gerade an seiner Spitze stattfinden? Aber die Frage ist leicht $z u$ beantworten. Der vom Oesophagus gegen die Trachea gerichtete bindegewebige $\mathrm{Zug}$, in welchem in der Längsrichtung reichliche Blut- und mit ihnen jedenfalls auch Lymphgefässe verlaufen, stellt eine Bahn dar, auf der Entzündungs-Processe 
bronchialer Lymphdrüsen sich leicht woiter fortsetzen und so ev. bis zum Epithel gelangen können. Daher finden wir auch schon im nicht narbig veränderten, subepithelialen Bindegewebe geringere Kohlemengen. Aber auch die Bildung kleiner Lymphdrüsen, in denen die Entzündungen sich zunächst localisiren, ist unter den abnormen Bildungsvorgängen weiter als sonst gegen den Oesophagus ausgedehnt. Neben jenem bindegewebigen Strang liegen, wie aus mehreren der unter 2 und 5 besprochenen Objecte hervorgeht, bis in die Nähe der Musculatur kleine Drüsen, die im entzündeten Zustande mit ihm verwachsen können. Für diese Verhältnisse liefert ein nur makroskopisch untersuchtes Object eine weitere gute Illustration. Drei miteinander zasammenhängende, stark vergrösserte, also nicht geschumpfte, pigmentirte, aber nicht indurirte Lymphdrüsen liegen der Länge nach nahe am Oesophagus. Das Divertikel geht über der mittleren $a b$, und der von ihm seinen Ursprung nehmende Strang verläuft neben der oberen Drüse und legt sich ihr dicht an.

Es sind also alle Bedingungen gegeben, welche das Hinzutreten eines Entzündungs-Processes zu dem Divertikel verständlich erscheinen lassen.

Aber so begreifen wir os auch, weshalb nicht jede, weun auch noch so hochgradige, tubereulös-anthracotische Veränderung der zwischen Speiseröhre und Trachea gelegenen Drüse zu einer Verlöthung mit ersterer führt. Dazu sind besondere Bedingungen erforderlich, und diese sind eben in congenitalen Anomalien, in jenem bindegewebigen Zuge gegeben, der an der Wand des Oesophagus, bezw. an einem Divertikel sich inserirt. Fehlt er, so bleibt im Allgemeinen die Speiseröhre über den veränderten Drüsen verschieblich. Sie verwächst dann mit ihnen nur selten.

Auf diese Wetse können alle Tractions-Divertikel unter den gemeinsamen Gesichtspunkt der congenitalen Anlage gebracht werden. Ein Entzündungs-Process aber, der zu einer bestehenden Ausbuchtung hinzutrit, kann sie bei narbiger Schrnupfung vertiefen. Seltener ist es jedenfalls, dass er das eigentliche Divertikel erst schafft, dadurch, dass er auf jenen congenital angelegten Bahnen die Oesophagus-Wand erreicht und sie bei seiner Retraction mit sich zieht.

Die Gründe, welche für meine Auffassung maassgebend sind, 
mögen schliesslich noch einmal zusammengefasst sein. Erstens finden wir die gewöhnlich als Veranlassung zur Divertikel-Bildung vorausgesetzten primären Verlöthungen der Oesophagus -Wand mit Entzündungs-Heerden, insbesondere mit Lymphdrüsen nur selten, jedenfalls nicht entfernt so häufig, wie es bei der grossen Zahl der Divertikel der Fall sein müsste. Zweitens sind Divertikel, denen jede Beziehung zu einem entzündlichen Process fehlt, sehr häufig. Sie können nur aus Entwicklungs-Störungen abgeleitet werden. Drittens zeigen die meisten mit Narbengewebe in $\mathrm{Zu}$ sammenhang stehenden Divertikel Verhältnisse, welche sich nur unter der Annahme erklären lassen, dass zuerst eine Ausbuchtung vorhanden war, zu der dann ein Entzündungs-Heerd in Beziehung trat.

Die Benennung "Tractions-Divertikel" behält aber unter allen Umständen ihre Berechtigung. Die Ausbuchtung entsteht ja oder wird vergrössert durch den Zug, den der zwischen Speiseand Luftröhre ausgespannte Strang ausübt. Sie kann ausserdem durch narbige Retraction hinzutretender Entzündungs-Producte verstärkt werden. Die congenitale Anlage aber theilt das Tractions- mit dem Pulsions-Divertikel.

\section{III. \\ Casnistische Beiträge zur Kenntniss der Abdominal-Cysten.}

(Aus dem Pathologisch-anatomischen Institut in Bern.)

Von

Dr. med. Ernst Hedinger.

(Hierzu Taf. I.)

Die Genese der Abdominal-Cysten ist trotz zahlreicher casuistischer Mittheilungen der letzten Jahre immer noch sehr dunkel. Wir hoffen durch die Publication von 4 Fällen einen neuen Beitrag zur Klärung dieser Verhältnisse zu liefern. Wir können uns allerdings nur in 2 Fällen über die Genese der Cysten be- 


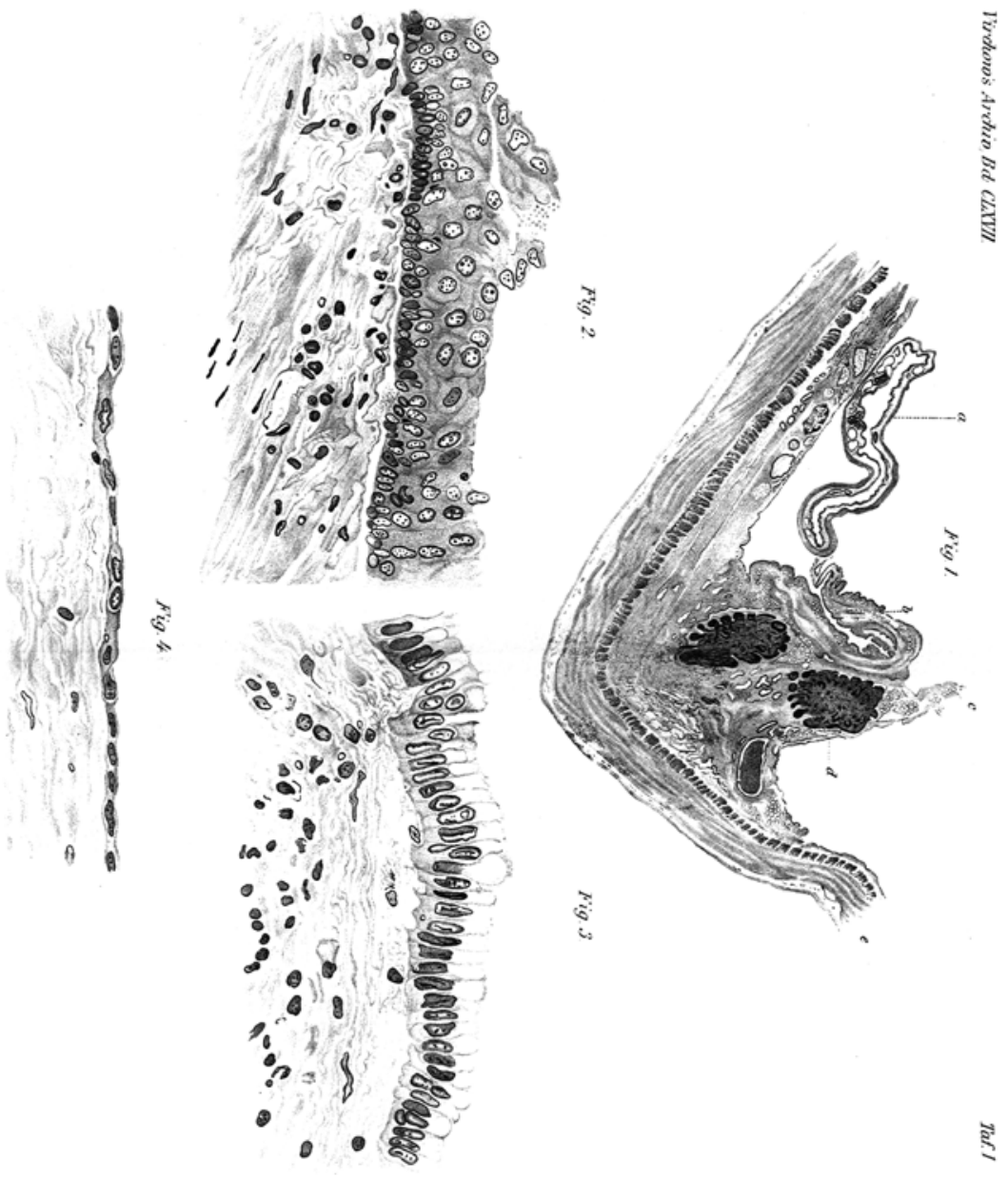

